\section{Visión Electrónica \\ Más que un estado sólido}

http: revistas.udistrital.edu.co/ojs/index.php/visele/index

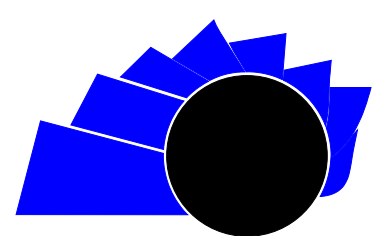

VISIÓN ELECTRÓNICA

A RESEARCH VISION

\title{
Genetic algorithms for optimization and study transport tours
}

\begin{abstract}
Algoritmos genéticos para optimización y estudio de viajes en transporte
\end{abstract}
María C. Parra Romero. ${ }^{1}$, Jaime Francisco Pantoja Benavides. ${ }^{2}$, Frank N. Giraldo Ramos. ${ }^{3}$

INFORMACIÓN DEL ARTÍCULO

\section{Historia del artículo:}

Enviado: 13/09/2016

Recibido: 02/04/2017

Aceptado: 22/08/2017

\section{Keywords:}

Genetic algorithm

Genetic operators

Route optimization

Traveling salesman

\section{Open access

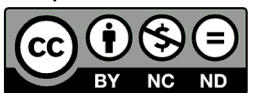

Palabras clave:

Algoritmo genético

Operadores genéticos

Optimización de ruta

Vendedor ambulante

\section{ABSTRACT}

This paper is the result of a research project developed by the DIGITI's research group at Francisco José de Caldas University, on optimization problems by using artificial intelligence and it shows the implementation of a genetic algorithm (GA) as a tool for planning and optimization transport tours, with the goal of finding the best path destinations for a fleet of vehicles. It presents basic concepts of the theory and the results obtained, about the administration and logistics in the supply chain, through a planning solution that optimizes the use of transportation resources.

\section{RESUMEN}

Este artículo es el resultado de un proyecto de investigación desarrollado por el grupo de investigación de DIGITI en la Universidad Distrital Francisco José de Caldas sobre problemas de optimización con inteligencia artificial; muestra la implementación de un algoritmo genético como herramienta de planificación y optimización de viajes en transporte, con el objetivo de encontrar la mejor ruta de destinos para una flota de vehículos. Presenta los conceptos básicos de la teoría y los resultados obtenidos, sobre la administración y logística en la cadena de suministro, a través de una solución de planificación que optimiza el uso de los recursos de transporte.

\footnotetext{
${ }^{1}$ BSc. In Industrial Technology, Universidad Francisco José de Caldas, Colombia. Current position: analyst Roldán Logística, Bogotá Colombia. E-mail: mcparra@roldanlogistica.com.

${ }^{2}$ BSc. In mathematics, Universidad Francisco José de Caldas, Colombia. Current position: Professor and researcher of DIGITI's research group at Faculty Technology, Universidad Francisco José de Caldas, Colombia. E-mail: jfpantoja2001@yahoo.es.

${ }^{3}$ BSc. In Electronic Control and Instrumentation, Universidad Francisco José de Caldas, Colombia. Current position: member of the DIGITI's research group at the Faculty Technology. E-mail: fngiraldor@udistrital.edu.co.
} 


\section{Introduction}

Transport is one of the primary activities in the supply chain, the transport of goods and services can be represented between one and two thirds of total logistics costs for companies [1]. From there, the importance of good planning and control. Traditionally, the study of optimization problems on spatial distribution and displacement has been addressed by dynamic programming techniques [2,3] and Operations Research $[4,5]$ and it is commonly known problem like the TSP (Traveling salesman problem), CPP (Chinese postman problem) or VRP (Vehicle routing problem), [6-8]. This article focuses on the application of genetic algorithms (GAs) to find the best optimization strategy for the problem of finding the shortest distance from the transport tour between different destinations or cities, considering also the minimum number of vehicles, in this case for a fleet of vehicles in the region of Cundinamarca.

From this perspective, this goal has been proposed by various heuristic optimization algorithms, in which we can highlight one, it is the GA $[9,10]$. The idea is that there is no guarantee of reaching the best solution and the most optimal solution will be available. Planning and management of distribution networks in supply chains requires the availability of efficient transport route, also its optimization and size of the vehicle fleet. The system tours optimization, not only affects the development of the company's operations, but also tactical and strategic decisions as the optimal size of the fleet, transportation costs estimate, schedule vehicle, maintenance, fuel management, conductor schedule management, failures or delays in cycle of cargo shipment, wrong delivery, etc. During the last decade, numerous applications have been oriented artificial intelligence techniques to a variety of technical and scientific fields, showing its efficiency, especially in the optimization problem like route planning case. GA power comes from the fact that it is a robust technology and that can successfully treat a variety of problems from different areas, including those in which other methods have drawbacks. While there is no guarantee that the GA finds the optimal solution, there is an empirical evidence that they are optimal level solutions in a time competitive with other optimization methods.

The GA, are adaptive and stochastic techniques (not deterministic ones), they inspired or emulate the mechanism of evolution, natural selection and genetic inheritance of individuals [11]. It can then be applied to converge a solution, transmitting the best solutions found by evolution between generations, reaching optimal.
That is, those individuals in the population with less suitable characteristics (as determined by their environment), more likely to disappear than those with characteristics adapted those most probabilities to survive [12].

The GA are implemented and are based on an initial set of random solutions called population. The information for each individual in the population is called chromosome, representing a solution of the problem to be optimized. Also a chromosome to be processed in the GA, besides, it can be encoded in binary or other numeric format. The generations evolve through successive iterations, by genetic operations between chromosomes of population individuals. During each generation, individuals are evaluated by using some measure of adaptation and the best ones are selected to create the next generation and so on, until the problem converges to an optimal solution or global minimum. Operations can be introduced randomly and their mutation of chromosomes code arbitrarily chosen as random factor that can help to accelerate the final convergence value.

\section{Methodology}

In the development of the solution to the problem of tour optimization, the GA was implemented in the Matlab $\AA$ software, taking the following steps:

\subsection{Encoding and initial population generation:}

Typically the initial population were randomly defined. It can also be obtained as a result of any additional algorithm, technique or local optimization heuristic, which can accelerate the convergence of GA. However, in some cases the disadvantage turns out, the premature convergence of GA solution, leading to local optima. Another aspect to consider is related to the ideal size of the initial population. It is intuitive to observe that small populations are at risk of not adequately cover the search space, while working with large populations can lead to problems related to the excessive computational cost and therefore, it speeds of convergence of the solution.

The individuals in the population correspond to each of the random tours to be defined previously, it is according to the size of the vehicle fleet. The chromosomes of individuals, are the set or vector data, which represent the sequence in which they connect cities on the tour and the each city correspond to genes in the chromosome. It should be noted that the sequence 
between cities raised must correspond to a path or Hamiltonian cycle [6], ie, not allowed to visit a city more than once and the path obtained should include them all. Then, each individual is represented by a numeric value in decimal, consisting of the coded sequence or chromosome, forming a vector whose length should be sized the same size for all individuals in the population.

First, each of the cities they must pass the vehicle fleet was codified in coordinates $(x, y)$, covering the geographic region of Cundinamarca with an area of 100 by 100 kilometers and causing an array of two coordinates, by the number of cities in the case is forty $(2 \times 40$ matrix size). Each index of the array (position of the city in the coordinate matrix) corresponds to a gene. A Chromosome would be the path or sequence of several genes that constitute an individual. The population, are formed by the tours or by many different individuals.

As an illustrative example, we defined a random initial population of size one hundred individuals. Shown the result of chromosomic encoding for one of the individuals (or gene sequence indexes of destination cities between 1 to 40) for a fleet of two vehicles:

Vehicle route 1: (1 133335172952016324143128 $\left.8 \begin{array}{lllllllllll} & 0 & 0 & 0 & 0 & 0 & 0 & 0 & 0 & 0 & 0\end{array}\right)$

Vehicle route 2: (2 25 26 37 36 611271092430157 2338318193439402122 12), see Figure 1

Figure 1: Example encoding chromosomes.

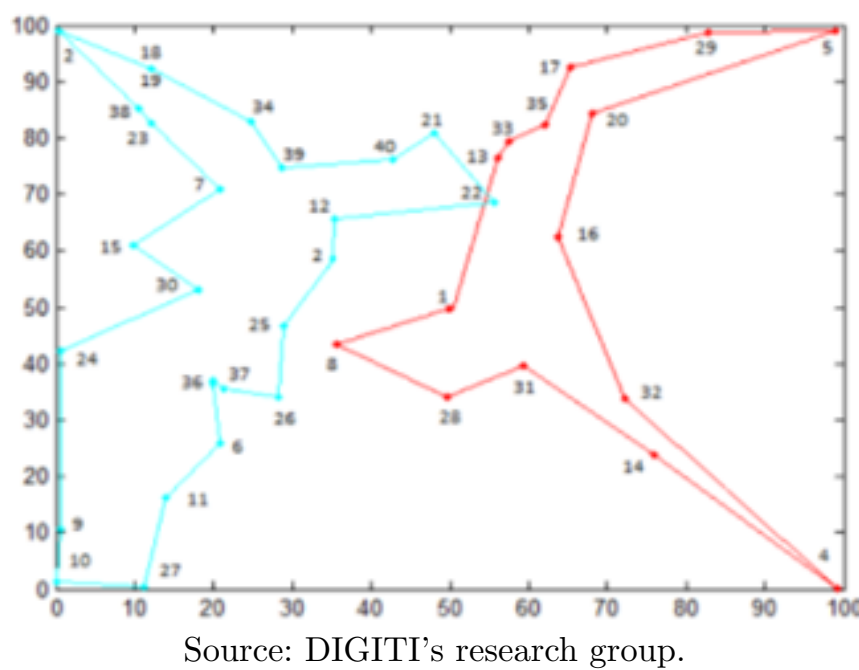

\subsection{Performance evaluation of individuals:}

All individuals (tours) of the population that were raised initially, should be evaluated by an evaluation function to determine its quality and rate their degree of specific setting (fitness). Which consists in this case, the cost function evaluation of the sum of each of the distances between the $\mathrm{n}$ cities of the tour, to determine which sequences are better adapted according to their score. Then, to verify the quality of each individual, making the sum of the distance between the coordinate point of the current city $\left(x_{i}, y_{i}\right)$ and the next city $\left(x_{i+1}\right.$, $\left.y_{i+1}\right)$ with all $n$ cities of each chromosome and thus yields the total length of each of the paths discussed:

\section{Sumofdistancecoordinates $(x, y)$ of cities}

For example, for the path of the first vehicle, the destination 1 to destination 13 is 23.81 kilometers, the target destination 13 to 33 is 5.27 kilometers, and so on. The sum of all distances between cities determines the total length of this path. The sum of the total distance between the paths of the two vehicles show adapting this proposed solution.

\subsection{Generation of new generations:}

It creates a new population of individuals through genetic operators, first you must select the best individuals for reproduction whose performance was evaluated in the previous step and which were made crossover operations or crossover and mutation.

\subsection{Selection of individuals:}

The primary way that useful information is transmitted, is that those individuals better adapted and with better quality factor, are more likely that are reproducing and their quality traits are preserved. Elitism selection by [13], aims to transmit and retain those characteristics fittest individuals along each iteration or generation of the genetic algorithm. Additionally, it also seeks to prevent the destruction of some of the best solutions and their characteristics, which may be caused by the crossover and mutation operators.

To implement the operator selection, tournament deterministic was performed [14], wherein it is stated that to make the selection of the best individuals to cross (elite method) should be performed tournaments between individual pairs of randomly selected and the alternative high quality factor (whose value is optimum in the objective function, ie shorter path) is selected to spend their genetic copy to the next generation. The number of alternatives and powers among couples tournament, depends on the size of the population. Then, 
if the population size is $n, 2 n$ tournaments be performed to form the total number of individuals.

The procedure is then to take a couple of individuals and calculate separately in each one, the total length in kilometers according to the tour sequence is as follows:

Individual 1: (1 23456789 )

Individual 2: (5 46921783 )

According to the cost evaluation function of distances between cities for the individual or path 1 , the total lenght would be 370 kilometers, while for the individual or path 2, is 460 kilometers, so the first individual is the winner and is selected for the population, it is made by genetic operations.

\subsection{Crossing individuals:}

The crossover operator to duplicate those individuals that have already been selected as the best in the population and to mix them to create a better generation of individuals, including those who have best fit or quality factor of the original population. In these cases, we used the Partial cross-Mapped Crossover PMX [15], which consists on defining two cutting points in the sequence of the chromosome, to initiate the crossover between those segments of genes, then undergo a validation of the route of descendents obtained, making a repairing of bad crossings or legitimizing of the secuence mistaken. This can be understood as a recombination of permutations which guarantees that each city or gene is not repeated in the chromosome of each offspring, ie are not repeated destinations on the same route.

PMX the crossover operator creates the next generation or offspring (solutions). For example, it considers the following routes or chromosomes as parent 1 and parent 2 respectively:

Parent 1: (1 23456789$)$

Parent 2: (546921783)

First, two cutting points are selected like crossover points XP1 and XP2 with random uniform probability along the sequence representing the route from each parent. Suppose that the first cut point is selected between the second and the third element of the tour, and the second between the sixth and the seventh element:

Parent 1: (1 $2|3456| 789)$
Parent 2: (5 $4|6921| 783)$

In the next segment below, there are some exchanged between parents, the path segments that were a product of the cuts. Thus, the path sequence of the first parent is copied to the second child. Similarly to the path sequence from the second parent is also copied to the first child, yielding:

Child 1: $\left(\begin{array}{ll|llll|lll}1 & 2 & \mathbf{6} & \mathbf{9} & \mathbf{2} & \mathbf{1} \mid & 7 & 8 & 9\end{array}\right)$

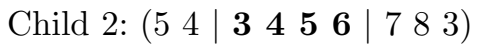

Then, this determines the transfer relations between resulting chromosomes. Cities or genes 1, 2, and 9 are duplicated in child 1 , while cities or genes 3,4 , and 5 are not present on this chromosome:

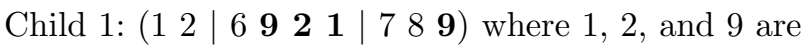
repeated 3,4 and 5 are missing

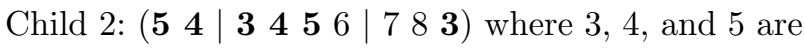
repeated, and 1, 2 and 9 are missing

According to the transfer relations determined above, cities repeated 1,2 , and 9 should be replaced by the cities missing 3, 5, and 4 respectively, while maintaining the route segment to be exchanged without change from baseline: see Figure 2.

Figure 2: Transfer Relations for repair operation.

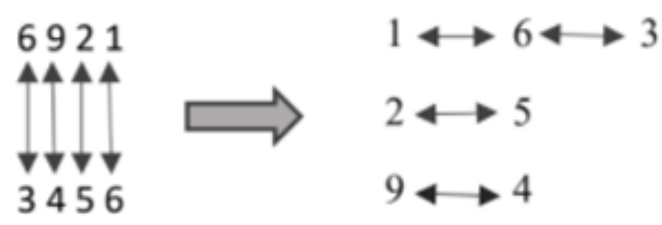

Source: DIGITI - s research group.

Finally, we should legitimize the children by using the transfer relations, ie to perform the repair of repeated or missing genes interchangeably according to transfers established relationships in the previous step:

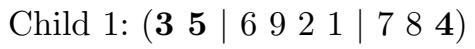

Child 2: (2 $9|3456| 78$ 1)

\subsection{Mutation of individuals:}

The mutation operator is taken into account, which provides a random element in the environment of the 
population of individuals. So what is sought, is to include a random factor that enables the reproduction of mutated individuals, although are not very well adapted, it may contain some useful information to explore other solutions in subsequent generations. While the crossover operator allows for the search along the space of possible solutions, the mutation operator increases its importance as the population of individuals are converging to a solution, since its aim is to produce new solutions from modification of a number of genes of the existing solution, with the intention of encouraging the variability within the population and avoid local minima convergence.

The mutation operator, should apply only to small percentage of individuals randomly selected of the population. For the case was implemented, the operator of reverse sequence mutation RSM [16], that involves taking a sequence within the chromosome, delimited by two cutting points MP1 and MP2 randomly chosen, such as MP1 $¡$ MP2. Then, the order of the sequence of genes enclosed within these cutoffs are reversed. It shows the application of RSM mutation operator, simply reverses the order of the sequence of a gene segment to be inserted again in the sequence of the tour:

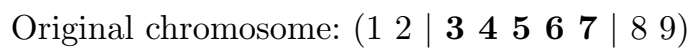

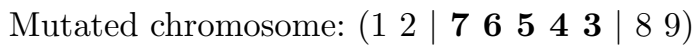

Figure 3: Genetic algorithm flowchart.

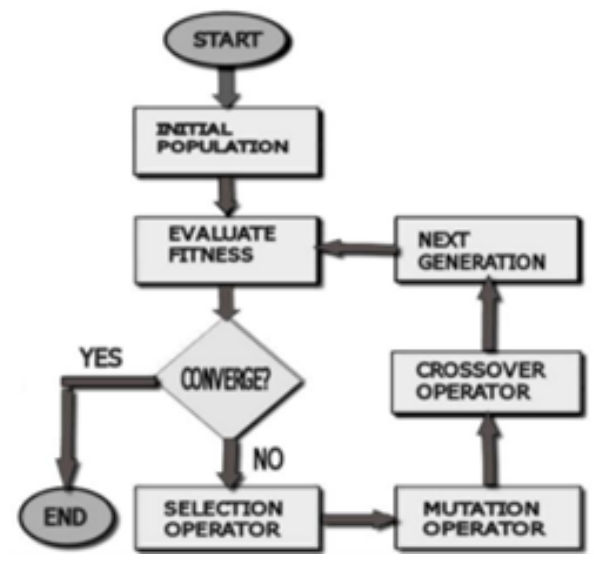

Source: DIGITI's research group.

\subsection{Algorithm's Finishing criterion:}

It iterates, with the new generation, discarding the old population by elitist succession and using the genetic operations explained above, for a given number of generations or until reaching the predefined termination criterion, or until that the global minimum is reached, ie the value of shortest-distance route. Although not all cases can guarantee that this solution is reached, the actual value would be the optimized. See Figure 3.

\section{Results}

Figure 4, shows the geographic region on which the study was carried out and optimization of transport tours, corresponding to the central geographic region over an area of $100 \times 100$ kilometers are also shown whereby cities must pass the route. We implemented the algorithm previously proposed as a script m-file in Matlab $\AA$, the spatial coordinates $(x, y)$ location of each city were entered in a matrix and were encoded as chromosomes by genetic algorithm. The city of Bogota was taken as the central point of supply through which must pass each vehicle routes as the fleet. Three scenarios were proposed as possible solutions with a fleet of two, three and four vehicles respectively.

Figure 4: Coding matrix destination cities over a region of 100x100 kilometers.

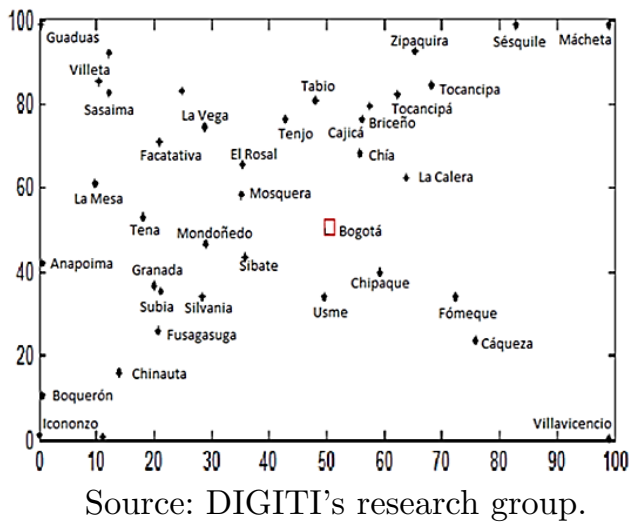

Figure 5: Route Optimization with GA for two vehicles.

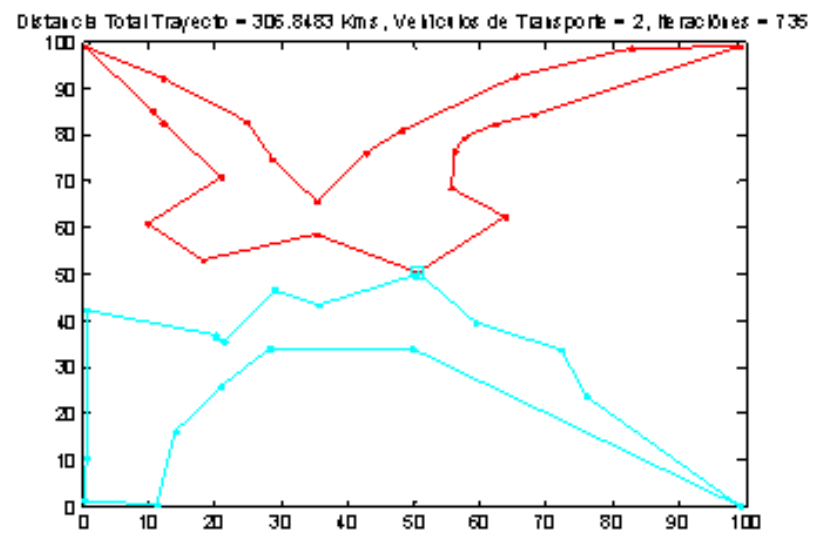

Source: DIGITI's research group. 
In the result of genetic algorithm optimization for a fleet of two cargo vehicles departing from Bogota supply center, it is evident that the total distance traveled by the two vehicles was 306.84 miles, additional routes are each one should perform. See Figure 5 .

In the case of employing a three vehicle fleet charging the total distance covered by them was 238.51 kilometers. See Figure 6.

Figure 6: Route optimization with GA for three vehicles.

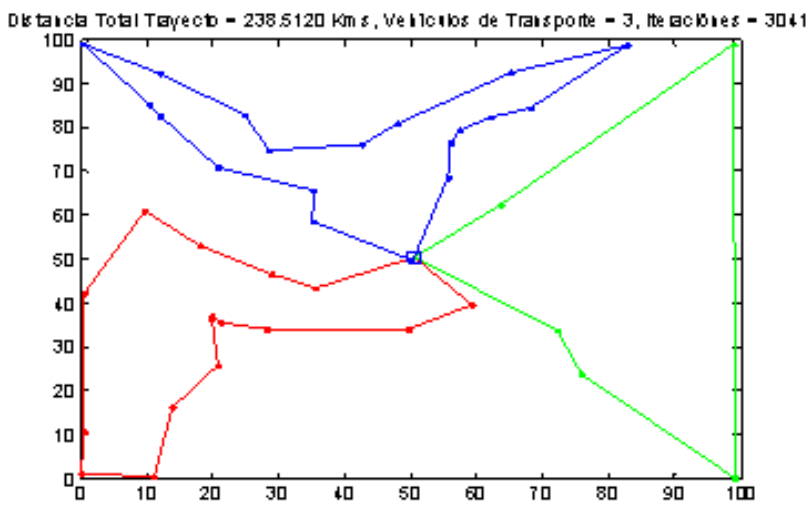

Source: DIGITI's research group.

And with a fleet of four vehicles, the result was a distance of 178.05 kilometers. (Figure 7).

It then verifies that the carrier given for these routes, the best scenario from the perspective of minimum distance tour is to use a fleet of four vehicles, according to the routes calculated by the genetic algorithm.

Figure 7: Route optimization with GA for four vehicles.

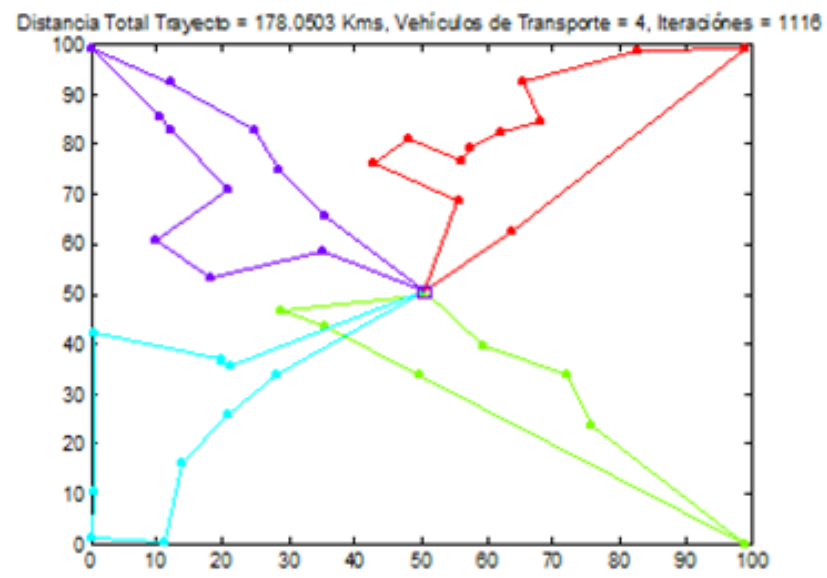

Source: DIGITI's research group.

\section{Discussion}

The costs associated with managing cargo transport logistics depend among various aspects on the ability to optimize time, transportation routes and distribution to the consumer or end. Under this premise, the scope of the results is to evidence a final strategy savings per kilometer of route optimization but also its size of the transport fleet.

The unit mileage costs is defined to estimate the margins, we estimated the cost per route, in order to define the freight costs for each alternative. In each of the scenarios studied, also minimizing the total service time by reducing the distances traveled in each route, their influences over the routes, the amount of gallons per kilometer consumed by vehicles, the tires cost, lubricants including maintenance, etc. Since on Route 1 a year is about $92,000 \mathrm{~km}$, while in route two is $71,400 \mathrm{~km}$ and $53,700 \mathrm{~km}$ route three respectively.

After completed the number of iterations in each simulation with the proposed scenarios, calculations were made to evaluate costs and make the best choice decisions.

Like it is shown the Figure 8, the total cost for the path 1, it was $\$ 81.050$ thousand of Colombian Pesos, the cost for the path 2, was $\$ 81.450$ thousand of Colombian Pesos and the cost for the path 3, was $\$ 83.350$ thousand of Colombian Pesos. It is evident, that for this case that the size of the fleet of cargo vehicles increases, the total distance decreases but drivers salary increases greatly.

Figure 8: Costs/tour options.
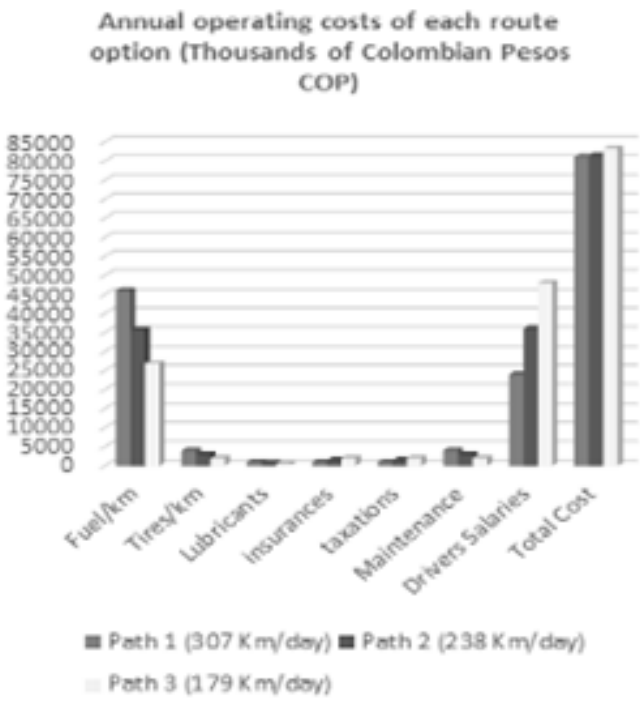

Source: DIGITI's research group. 
Therefore, the optimal solution from the point of view of total costs, was the tour 2, despite the fact that is not the tour with the lowest total distance, it is cheaper in its total cost, of course it was given the size of the fleet with respect to tires costs, maintenance, drivers' salaries, etc.

It can guide the future work, considering a GPS location information, road congestion, road speed limit, etc, all in real time for simultaneously redefining the appropriate route.

\section{Conclusions}

Although genetic algorithms do not ensure that solutions are reached, in terms of the global minimum, if it is possible to find a set of optimal solutions (minimum distance routes), which provide a good performance in comparison with solutions obtained by other methods.

Selection operators (deterministic tournament), crossover (PMX operator) and mutation (RSM operator) used, have shown good performance during the iteration of the algorithm and it always walked to optimal fast convergence of the solutions.

The first consideration when creating a GA is to define a representation of the problem. You must define the objective function, taking into account that you can achieve greater fitness (fitness) and truly can give a better solution to a given problem. Another common problem is that the GA may tend to premature convergence to a local minimum, due to the fact that this following an incorrect definition of the initial population. The GA work particularly well solving problems where the potential solution space is too large for an exhaustive search in a reasonable time, as in this case of optimizing transport routes. The most important feature of using the GA, is the evaluation of scenarios by using simulation techniques that highly allow to recreate the possible solutions.

It was evident, the potential of GA in its implementation in logistics problems, as in the case of route planning, with good results as an optimization method facilitating decision-making in this field.

\section{Aknowledgments}

To the DIGITI group, for funding research projects.

\section{References}

[1] H. Ronald, "Logistics Management Supply Chain". Fifth Edition, Mexico: Pearson Education, 2004, p 14 and 64 .

[2] M. Held and R. M. Karp, "A dynamic programming approach to sequencing problems," Journal of the Society for Industrial and Applied Mathematics, issue 10,1962, pp. 196-210

[3] R. Bellman and S. Dreyfus, "Applied Dynamic Programming". Princeton University Press, 1962. https://doi.org/10.1515/9781400874651

[4] Winston, "Operations Research W.L. Applications and Algorithms". Editorial Latin America, 1994.

[5] M.M. Flood, "The traveling salesman problem", Operations Research, vol 4, no. 1, Feb. 1956. https: //doi.org/10.1287/opre.4.1.61

[6] K. Menger, "Das Botenproblem," Mathematische eines Ergibnisse Kolloquims 2 Teubner, Leipzig, pp. 12-12, 1930.

[7] J. B. Robinson, . ${ }^{\circ}$ the Hamiltonian game (a traveling-salesman problem)", RAND Research Memorandum RM-303. 1949.

[8] G. Dantzig, R. Fulkerson, S. Johnson, "Solution of a Large-Scale Traveling-Salesman Problem" Journal of the Operations Research Society of America, vol 2, no. 4 p. 393- 410, Nov, 1954. https://doi.org/10. 1287/opre.2.4.393

[9] Donald Davendra and Yuan-bin Mo "The Advantage of Intelligent Algorithms for TSP", Traveling Salesman Problem, Theory and Applications, Prof. Donald Davendra (Ed.), InTech, 2010, https://doi. org/10.5772/12897

[10] T. Murata, and H. Ishibuchi, "MOGA: Multi-objetive genetic algorithms", Proceedings of the second IEEE International Conference on Evolutionary Computation 1, pp. 289- 294, 1995. https://doi.org/10.1109/ICEC.1995.489161

[11] J.H Holland, "Adaptation in natural and artificial systems", MIT Press, 1975.

[12] C. Darwin, "The origin of species by means of natural selection" New york: D. Appleton and Company, 1859.

[13] B. Chakraborty and P. Chaudhuri, . ${ }^{\circ} \mathrm{n}$ the Use of Genetic Algorithm with Elitism in Robust and Nonparametric Multivariate Analysis", austrian journal of statistics, issue 32, pp. 13-27, 2003. 
[14] D. E. Goldberg and K. Deb, "comparative analysis of selection schemes used in genetic algorithms". In G. J. E. \& Rawlins (Eds.), Foundations of genetic algorithms, Morgan Kaufmann Publishers, pp. 307-333, 1991. https://doi.org/10.1016/ B978-0-08-050684-5.50008-2

[15] D. E. Goldberg and R. Lingle. "Alleles, loci, and the traveling salesman problem". In Proceedings of the International Conference on Genetic Algorithms and Their Applications, pp.154-159, 1985.

[16] O. Abdoun, J. Abouchabaka and C. Tajani "Analyzing the Performance of Mutation Operators to Solve the Travelling Salesman Problem CoRR",IJES International Journal of Emerging Sciences, vol 2, no 1, pp. 61-77, 2012. 